Wie wir dem Schlusse der Einleitung genannten Buches entnehmen, beabsichtigt der Graf ausführlichere Berichte über die seltenen und ungewöhnlicheren Erscheinungen in der Vogelwelt Galiziens diesem Cataloge folgen zu lassen, die hier des Raumes wegen nur kurz Erwähnung finden konnten, und die das hier vorliegende Material wesentlich $\mathrm{zu}$ bereichern versprechen.

Allen, welche sich für die Ornis Galiziens interessiren, können wir Graf W. Dzieduszycki's Catalog, welcher die beste Uebersicht über die Vogelwelt dieses Landes gewährt, wärmstens empfehlen, und wir hegen die Ueberzeugung, dass ihn Jeder mit derselben Befriedigung aus der Hand legen werde, wie wir es gethan.

Villa Tännenhof b/Hallein, im Februar 1882.

v. Tschusi zu Schmidh offen.

\title{
Ueber eine kleine ornithologische Sammlung von den Philippinen.
}

Von

Dr. Kutter in Neustadt O/Schl.

Unsere bisher ziemlich eingeschränkte Kemtniss der philippinischen Ornis hat in den letzten Jahrzehnten, vorzugsweise durch eine Reihe werthroller Arbeiten des leider inzwischen verstorbenen Marquis of $\mathrm{T}$ weeddale (Lord $\mathrm{W}$ alden) eine wesentliche Bereicherung erfahren. Diese in verschiedenen wissensebaftlichen Zeitschriften zerstreuten Arbeiten des ausgezeichneten englischen Forschers gesammelt und vor Kurzem herausgegeben zu haben, ist das Verdienst eines Neffen desselben: Mr. W a r d law Ramsay. $\mathrm{Zu}$ bedauerı bleibt beiläufig, dass der stattliche Quartband, dessen Einsicht mir von befreundeter Seite ermöglicht wurde, nicht im Buchhandel erschienen, sondern nur der Privatvertheilung vorbehalten ist. - In einem Anhange des Sammelwerkes hat sich der Herausgeber der dankenswerthen Mühe unterzogen, eine übersichtliche Zusammenstellung aller z. Z. bekannten philippinischen Vögel, nach ihrer geographischen Vertheilung auf den einzelnen Inseln und ihrem etwaigen Vorkommen zugleich auch in den benachbarten Gebieten, zu liefern. Diese Liste weist die relativ beträchtliche Anzabl von 379 Arten anf, deren 19 indessen dem Verfasser aus verschiedenen Gesichtspunkten zweifelhaft erscheinen. 
Dass die hier angegebene Zahl von Philippinen-Vögeln (eingeschlossen werden in das Gebiet: Palawan, Balabac und der Sulu-Archipel) sich in der Folge durch eingehende Forschungen noch vermehren wird, kann mit Bestimmtheit erwartet werden. Dennoch dürfte es wohl nur einem besonders glücklichen Zufalle zuzuschreiben sein, wenn die so wenig umfängliche, noch dazu von einem Nicht-Ornithologen und fast ausschliesslich auf der verhältnissmässig am meisten durchforschten Hauptinsel zusammengebrachte Collection, über welche hier kurz berichtet werden soll, mehrere Arten enthält, welche entweder überhaupt, oder doch für das specielle Gebiet neu erscheinen.

Der Sammler, Herr Otto K och aus Altona, kehrte im Sommer d. J. (1881), nach mehrjährigem Aufenthalte auf den Philippinen, zu kurzem Besuche in die Heimath zurück. Von dem regen Interesse desselben für Naturwissensehaften und seiner vielseitigen sammlerischen Thätigkeit, neben der Erfüllung von Berufspflıchten, legen besonders die mitgebrachten werthvollen Collectionen entomologischer, anthropologiseher und ethnographischer Objecte Zeugniss ab. Das beschränlkte ornitbologische Material wurde nur nebenher gesammelt; ich erhielt dasselbe durch gütige Vermittelung eines schon früher zurückgekehrten Freundes und Reisegenossen des Sammlers, Herrn Dr. A. Schadenberg. Die kleine Collection umfasste, neben einer Partie Bälge und Spirituspräparate von Vögeln, auch eine Anzahl von Nestern und Eiern, welche letzteren leider, durch ungeeignete Verpackung, grössentheils stark beschädigt in meine Hände gelangten. Noch bedauerlicher ist es, dass den Eiern und Nestern zwar durchweg die tagalischen Namen der betreffenden Vögel, nur zum Theil aber diese selbst beilagen. Abgesehen von letzteren Eällen, konnte daher die Bestimmung ihrer specifischen Zugehörigkeit meist nur mit Wahrscheinlichkeit ausgeführt werden. Wenn ich trotzdem nicht Anstand nehme, dieser mehr minder unsicher identificirten Objecte in der Folge zu erwäbnen, so geschieht dies theils aus dem Grunde, weil äber die Fortpflanzung philippinischer Vögel bisher nur wenige Notizen vorliegen, sodann aber in der Voraussetzung, dass die beigefügten tagalischen Bezeichnungen früher oder später einen Anhalt zur Controle der diesseitigen Annahmen gewähren ditrften.

Was die Bestimmung der Vögel anlangt, so ist diese, - mit der allzeit bereitwilligen und bewährten Unterstützung des Herausgebers dieser Zeitschrift, dem ich dafür zu besonderem Danke 
verpflichtet bin - durch Vergleichung von Exemplaren des Berliner Museums erfolgt, welches auch einen Theil der Objecte erworben hat; ich werde diese nachstehend bezeichnen und hoffe, ein eingehenderes Referat über mehrere derselben aus berufenerer Feder in Aussicht nehmen zu dürfen. Eine gewisse Schwierigkeit hinsichtlich der exacten Feststellung der Provenienz der vorliegenden Bälge schien sich anfänglich aus dem Umstande zu ergeben, dass den in der überwiegenden Mehrzahl von Herrn Koch persönlich gesammelten Vögeln noch eine kleine Partie solcher beilag, welche von dem Genannten gelegentlich in Manila, aus dem Nachlasse eines Sammlers, erworben wurden und hinsichtlich deren die Feststellung der Species grösstentheils, wenn nicht aussehliesslich, aut Celebes als Sammelgebiet zu verweisen schien. Glücklicher Weise gelang es nachmals, abgesehen von mündlichen und schriftlichen erläuternden Angaben des Herrn Koch, diese beiden Kategorien durch vergleichende Prüfung gewisser Eigenthümlichkeiten der Präparationsmethode (welche bei den Luzon-Bälgen zum Theil sehr zu wünschen lässt), mit Bestimmtheit von einander zu sondern, so dass hier, unter gewissenhafter Ausscheidung aller irgend welchem Zweifel Raum gebenden Exemplare, lediglich diejenigen Arten aufgefuhrt sind, welche mit Sicherheit als auf den Philippinen, bezw. - mit einziger Ausnahme der auf Cebú erbeuteten Collocalia - auf Luzon gesammeit, recognoscirt werden konnten.

Endlich möchte ich diesen Vorbemerkungen noch die Mittheilung hinzufugen, dass die Herren Koch und Dr. Schadenberg im Herbst neuerdings nach den Philippinen abgereist sind, wo sich dieselben demnächst ausschliesslich der Sammlung naturwissenschaftlicher Objecte widmen werden und vorzugsweise Süd-Mindanao als Operationsgebiet in Aussicht genommen haben. Es ist zu erwarten, dass auch die ornithologische Ausbeute diesmal durch Sorgfalt der Präparation, Notirung von Maassen und Geschlecht der Individuen, Angabe der Fürbung unbefiederter Körpertheile im frischen Zustande etc. in erhöhtem Grade wissenschaftlichen Auîorderungen entsprechen werde und somit dem nachstehenden sehr fragmentarischen localfaunistischen Beitrage werthvollere folgen können. -

1. Calliope camtschatkensis (Gm.), 1 万; die carminrothen Federn der Kehle zeigen schmale, weissliche Spitzen.

2. Megalurus palustris Horsf., 2 Vögel, 1 Nest, Eier. Tagalischer Name "Tintirioc". Die von Bernstein (Jahrg. 1859, S. 261 d. J.) nach javanischen Exemplaren gegebene Beschreibung 
des Nestes und der Eier stimmt mit den vorliegenden im Wesentlichen überein; dagegen haben Thienemann (Fortpflanzungsgesch. d. gesammt. Vögel) offenbar nicht Eier dieser Art, sondern solche einer Drymoica vorgelegen.

Das Nest ist ein ziemlich lose und unordentlich aus zerschlissenen Gras- oder Schilfblättern zusammengehäufter, tief napfförmiger Ban ; innen mit ähnlichem, aber feinerem Material ausgelegt. Höhe 10,5; Durchmesser 9-10; Tiefe des Napfes 7, oberer Durchmesser desselben $7,5 \mathrm{Cm}$.

Die Eier sind gedrungen oder etwas gestreckter eiförmig, auf weissem oder schwach röthlichgrauen Grunde meist dicht besprengt mit feinen und sehr feinen, rothbraunen, verwaschenen oder schärfer accentuirten Pünktchen und Fleckchen, die am stumpfen Ende gewöhnlich häufiger stehen, oder daselbst einen lockeren Kranz bilden. Unter dieser Oberzeichnung finden sich etwas grössere, grauviolette, verwaschene Schaalenflecke. Bei durchfallendem Lichte erscheint die Schaale schwach grünlich, mit durchscheinender Zeichnung. Das Korn ist fein und gleichmässig; Schaalenglanz gering oder mässig stark. Die Maasse schwanken zwischen $25,5+17,7$ und 22,5 + $17 \mathrm{Mm}$., das Gewicht zwischen 18,5 und 21,5 Cg. Der oologische Gesammttypus ähnelt sehr dem der Schwirrvögel-Gruppe, insbesondere Locustella fluviatilis; zugleich aber zeigt er auch mehr minder deutliche Annäherung an den der Pycnonotiden.

Ob einige ausserdem unter dem einheimischen Namen "Calaga" beiliegende und mit den eben geschilderten, bis auf etwas geringere Grössen- und Gewichtsverbältnisse, sehr übereinstimmende Eier gleichfalls der in Rede stehenden Art oder vielleicht dem verwandten Megal. ruficeps Tweed. angehören, muss ich dahingestellt lassen.

3. Ixos goicuier (Scop.) - ? Eine Anzahl unzweifelhafter Pycnonotiden-Eier der Sammlung, mit der tagalischen Bezeichnung "Ulaclac", bin ich geneigt, der bezeichneten und bekanntlich anf Luzon nicht seltenen Art zuzuschreiben, da sie in Nichts von den Eiern des nahe verwandten, von Einigen als identisch betrachteten Ixos analis Horsf., deren ich von Malacca empfing, zu unterscheiden sind. Maasse: $22,4+16,3$ bis $21,2+15,0 \mathrm{Mm}$. Gewicht: 13,5 bis $18 \mathrm{Cg}$. Alle übrigen Kennzeichen der schönen Eier entsprechen den bekannten der Gesammtgruppe.

4. Orthotomus Derbyanus Moore - ? Ein charakteristisches Schneidervogel-Nest mit 2 Eiern, unter der tagalischen Bezeichnung 
„Mananáje", gehört wohl unzweifelhaft dieser Species an, da eine andere verwandte Art bisber nicht auf Luzon beobachtet wurde. Das überaus zierliche beutelförmige Nestchen gleicht sehr den in dieser Zeitschrift beschriebenen von O. sexium Horsf. (Jahrg. 1859, S. 265) und O. longicaudus (Gm.) (Jahrg. 1855, S. 328). Die Grundlage desselben bildet ein Büschel herabhängender Blätter mit etwas wolliger Unterseite, deren Stiele oben, wo sie an dem Zweige festsassen, zusammenlaufen und deren Ränder, sowohl an der Rückseite des Nestes, als auch vorn, wo sie ziemlich weit auseinanderklaffen, mit vom Vogel selbst gesponnenen Baumwollenfäden zusammengeheftet sind. $\mathrm{Da}$, wo die $\mathrm{F}$ äden den Blattrand durchbohren, sind sie, behufs ihrer Befestigung an der oberen Blattseite, gleich einem Knoten, zu Klümpchen zusammengedreht. Der Grund des Nestchens ist nicht von den Blättern umschlossen, und um demselben mehr Haltbarkeit zu geben, sind hier, wie an der gleichfalls mehr freien Vorderseite, aussen einige feine Rispen und Hälmchen zusammengewebt. Innen ist das Nestchen dicht und gleichmässig mit feinster, seidig glänzender Saamenwolle ausgefüttert. Der Eingang befindet sich vorn und oben, zum Theil von den hier zusammenlaufenden Blättern überdeckt. Höhe des Nestes 10,5; grösste Breite 5,5 Cm.

Die Eier entsprechen dem Typus der Verwandten; sie sind gestreckt eiförmig, mit wenig verjüngter Spitze, also der Walzenform sich nähernd. Die Schaale ist glatt, mässig glänzend; das Korn sehr fein, mit vereinzelten flachen Poren. Der Grund erscheint rahmfarben, schwach röthlich gelb angehaucht, bei durchfallendem Licht ganz lell gelblichgrün; darauf stehen gelbrothe, meist etwas verwaschene Oberfleckehen und zwischen diesen am stumpfen Ende, wo sich die Zeichnung häuft und ein lockeres Kränzchen bildet, grauviolette Schaalenflecke. Dimensionen: $16,5+11,9$ und $16,7+11,7$; Gewicht: $7 \mathrm{Cg}$.

5. Rhipidura nigritorquis Vig.; tagalisch: "Maria-capri"; 1 Vogel, Nester, Eier.

Das Nest ist ein ebenso zierlicher als solider, anssen und innen sorgfältig geglätteter Napfbau, welcher auf einer Astgabel befestigt ist und dem der meisten Gruppengenossen ähnelt. Das Baumaterial haben hier vorzüglich Baststreifen und andere biegsame Pflanzentheile geliefert; die innere Auskleidung besteht lediglich aus feinen Gräsern, die Aussenseite ist überall sauber und ziemlich dicht mit Spinnengeweben überzogen. Höhe $7-7^{1} / 2$, 
Breite $61 / 2$, Tiefe des Napfes 3, oberer Durchmesser desselben $5 \mathrm{Cm}$.

Die Eier sind gedrungen oder etwas gestreckt eiförmig, von gelblicher Rahmfarbe, innen grünlich gelb durchscheinend. Die Zeichnung besteht in bräunlichen Ober- und grauen Schaalenflecken, welche meist in Form eines mehr oder weniger dichten Kranzes gegen das stumpfe Ende zu angeordnet sind und die übrige Oberfläche fast ganz frei lassen. Das Korn ist fein, die Schaalenoberfläche matt glänzend. Maasse: $16,6+13,8$ bis $19,6+13,7$; Gewicht: 9-14 Cg. Im Ganzen können die Eier als sehr vollkommene Miniaturausgaben derer von Lanius collurio, in verschiedenen Varietäten dieser Art, gelten, - wie denn ïberhaupt sämmtliche mir bekannte Eier von Rhipidura-Arten, sowie gewisser verwandter Genera keineswegs dem oologischen Typus der Muscicapinae, sondern in ausgesprochener Weise dem der Laniinnae entsprechen. Ich bin geneigt, hieraus zu schliessen, dass entweder die von einigen Systematikern beliebte weite Trennung der Lanizdae und Muscicapidae im System eine phylogenetisch nicht wohlbegründete ist, oder gewisse Genera der einen Gruppe naturgemässer in der anderen zu rubriciren sein würden - et vice versa.

6. Lanius cephalomelas Bp., tagalisch "Camisote"; 1 Vogel (z. Z. im Museum Berolinense - vergl. über das Expl.: Ornith. Centr. Bl. 1881, S. 147) 1 Nest mit 4 Eiern.

Das Nest ähnelt sehr dem unsers Lanius minor; es ist napfförmig, aussen von dürren Pflanzenstengeln und Baststreifen erbaut, denen hier und da, besonders an der Basis, Woll- und Baumwollflocken eingefügt sind; innen ist es mit feinen Hälmchen ausgelegt und sorgfältig geglättet. Höhe 8, Durchmesser 11,5. Tiefe des Napfes 4,5, oberer Durchmesser desselben $8 \mathrm{Cm}$.

Die vorliegenden Eier zeigen den bekannten Typus der Laniiden und sind von solchen des $L$. nigriceps Jerd. aus Indien nicht zu unterscheiden. Maasse: $22,5+18,6$ und $23+17,4 \mathrm{Mm}$; Gewicht: 20-22 Cg.

7. Broderipus acrorhynchus Vig. 1 Vogel (ठ ad.) 1 Nest mit 3 Eiern. Tagalisch "Guluayaban". Nach v. Martens (Jahrg. 1866, S. 16 d. J.) giebt schon Pater $\mathrm{Camel}$, zu Anfang des vorigen Jahrhunderts, den einheimischen Namen ähulich an ("Guliyavan") und Bernste in erwähnt (Jahrg. 1859, S. 270 d. J.), dass Oriolus indicus Briss. von den Javanesen $u$. a. auch „Pulavan“ genannt werde, zugleich führt er die Etymologie auf das Sanskrit zurück. 
Offenbar liegt hier in beiden Fällen derselbe Wortstamm vor ("Culay" tagalisch $=$ gelb).

Das Nest ist, ganz ähnlich denjenigen unseres Pirols, hängend in einer Astgabel erbaut. Baststreifen, welche zunächst um die Seitenzweige der letzteren geschlungen sind, bilden die Grundlage; damit sind alsdann andere biegsame Pflanzentheile, Baumwollenflocken, sowie auch einzelne Woll- und Baumwollenfäden, die der Vogel als geeignet befunden und irgendwo aufgelesen hat, verwebt. Innen ist der Napf mit feinen PHanzenstengeln ausgelegt und sorgfältig gerundet; aussen sieht der Bau ziemlich unordentlich und liederlich aus. Höhe 9, Längsdurchmesser 15, grösste Breite 13, Tiefe des Napfes 6, oberer Durchmesser desselben $10 \mathrm{Cm}$.

Auch die Eier stimmen durchaus mit denen von O. galbula überein, nur sind sie etwas grösser, besonders im Querdurchmesser, und dementsprechend auch schwerer. Maasse: $30,6+23$ bis $31+23,5 \mathrm{Mm}$.; Gewicht: $46-49 \mathrm{Cg}$.

8. Melanopitta atricapilla (Cuv.), 2 Vögel, deren einer als ơ vom Sammler bezeichnet ist. Nach Maassgabe der bei dem andern Exemplar völlig gleich weiten Ausbreitung des Weissen auf den Primärschwingen (cf. Twe ed dale, Proc. Zool. Soc. 1878, p. 949) würde auch dieser demselben Geschlechte angehören. Bei No. 1 zeigt sich das Grün der Brust ausgebreiteter und deutlicher mit Blau vermischt als bei No. 2, wo von dieser Färbung, wie bei zwei Zamboanga-Bälgen Tweeddale's (l. c.), kaum noch etwas zu bemerken ist. Es dürfte sich hierbei also wohl nur um individuelle, bezw. Altersverschiedenheiten handeln.

9. Erythropitta erythrogastra (Tem.), 1 o; in dem grünen Brustbande zeigen sich nur einige wenige blaue Federn und ist daher das vorliegende Individuum als ein jugendliches anzusprechen (cf. Tweed dale, l. c., p. 948).

10. Acridotheres cristatellus (Lin.), nur eine Anzahl Eier. Ueber den Vogel berichtet Herr Koch, dass derselbe von den Tagalen „Martini" genannt werde und die Species erst im Laufe dieses Jahrhunderts durch den Verein ,Amigos del pays" (Freunde des Landes) aus China auf Luzon eingeführt worden sei. Hier habe er sich rasch eingebürgert und so vermehrt, dass er jetzt zu den häufigsten Vögeln der Insel gehöre. Der Zweck der Einführung: Vertilgung der Heuschreckenschaaren, sei freilich unerreicht geblieben, da der Vogel, nach den eigenen Beobachtungen des Berichterstatters, sich dieses Geschäft nicht besonders angelegen 
sein lasse. - Die Tradition der Einführung des Vogels von China wird auch von anderen Autoren, so von Meyer und Swinhoe, erwähnt, doch müsste dieselbe um einen bedeutenden Zeitraum zurückdatirt werden, wenn v. Martens Recht hat, indem er diese Species (1. c., S. 14) mit dem „Kaaling" des Paters Camel indentificirt.

Die Eier ähneln, wie zu erwarten, ganz denen der nahen Verwandten, besonders Acrid. tristis; sie zeigen dieselben Variationen der Form, des Schaalenglanzes, des Korns und der Farbenintensität wie diese, übertreffen dieselben aber, nach den vorliegenden Stüeken, im Durchschnitt etwas an Dimensionen und Gewicht. Maasse: $30+20,2$ bis $33,7+22 \mathrm{Mm}$.; Gewicht: $41-60 \mathrm{Cg}$.

11. Gymnops calvus (Lin.), tagalisch "Goleto", eine Benennung, die neueren Datums sein muss, da sie von Camel (nach v. Martens, 1. c.) unter den durch ihn reproducirten Localnamen der Species nicht mit aufgeführt wird. 2 alte Vögel; ganze Oberseite grau. Nach einer Notiz des Sammlers: „Augen braun, Umfang über die Brust $16 \mathrm{Cm}$., Totallänge $28 \mathrm{Cm} . "$

12. Corvus philippinus Bp., 1 Vogel (Museum Berol.).

13. Munia Jagori Cab., 1 Nest, Eier. Der Vogel wird von Herrn Koch als „kleiner Fink von kaffeebrauner Farbe, mit schwarzer Brust und sehwarzem Kopfe" beschrieben; der tagalisehe Name ist "Mayan-pula" (mit "Maya" wird von den Tagalen Padda oryzivora bezeichnet, "pula" = roth). Die Zugehörigkeit von Nest und Eiern zu der genannten Speciès dürfte hiernach keinem Zweifel unterliegen, vorausgesetzt, dass $M$. minuta Meyen in der That nur das Jugendkleid von Jagori ist." )

Das Nest ist ein ziemlich lockerer und kunstloser Bau; die Grundlage besteht aus breiteren und schmäleren Gramineen-Blättern, auf welchen ein flacher, lose zusammenhängender Napf von feinen Hälmchen ruht. Die Construction ist einigermassen befremdend, da bekanntlich die meisten Verwandten unseres Vogels geschlossene Nester bauen. Höhe 6, Durchmesser $10^{1 / 2}$, Tiefe des Napfes $4 \frac{1}{2}$, oberer Durchmesser desselben $5 \mathrm{Cm}$. Die Eier sind, wie die aller

*) Die Vergleichung des Original-Exemplares der Fringilla minuta Meyen im Berliner Museum mit inzwischen daselbst eingetrofitenen jungen Vögeln von Jagori hat unzweifelhaft herausgestellt, dass mimuta Meyen der junge Vogel von Jagori ist.

Der Herausgeber. 
übrigen Spermestinae, rein weiss; Schaale schwachglänzend, feinkörnig; Gestalt normal eiförmig. Maasse: $16+11,1$ und $15,8+11,5$ Mm.; Gewicht: 5,5 Cg.

14. Merops philippinus Lin., 2 Vögel, ad.

15. Sauropatis chloris (Bodd.), 2 Vögel. Brust und Seiten des einen (jüngeren)Individuums hell-isabellgelblich, mit Spuren schmaler dunkler Querwellen, welche auf dem weissen Nackenbande, sowie an den Halsseiten intensiver und zahlreicher auftreten. Bei dem anderen Exemplar Unterseite weiss; nur am Nackenbande und den Halsseiten noch vereinzelte, schwachangedeutete dunkle Querwellen.

Zwei Eier, welche mit der Bezeichnung "Casai-casai" in der Collection vorliegen, können sehr wohl dieser Species angehören, da sie mit authentischen Exemplaren von anderer Provenienz auch in den Dimensionen sehr übereinstimmen. Sie sind, wie die Eier aller Alcedinidae und der verwandten Gruppen, reinweiss, glattschaalig, glänzend, gedrungen gleichhälftig, oder etwas ungleichhälftig, feinkörnig, mit nur schwach angedeuteten Poren. Maasse: $31,5+24,8$ und $20+25 \mathrm{Mm}$. (Das Gewicht kann wegen bedeutenden Schaalendefectes beider Stücke nicht präcisirt werden.)

16. Entomobia gularis (Kuhl), 2 Vögel, ad.

17. Astacophilus Lindsayi Cab., 1 o (Mus. Berol.).

18. Collocalia cebuensis, n. sp., tagalisch „Balin-sayao" (Höhlenschwalbe).

Am 6. März 1881 besuchte Herr Koch auf der Insel Cebú die Wohnhöhle einer Gesellschaft von Salanganen. Er fand die Vögel beim Brutgesebäft; jedes der in der bekannten Weise an den Höhlenwänden angeklebten Nester enthielt nach seiner Erinnerung drei Eier, welche bereits sämmtlich in der Entwickelung so vorgeschritten waren, dass sich ihre Präparation, bezw. Entleerung unmöglich erwies und die drei eingesandten, mehr minder defecten Exemplare in Spiritus conservirt werden mussten. Einen der abfliegenden Vögel fing der Sammler mit dem Schmetterlingsnetze; er wurde anfänglich gleichfalls in Spiritus aufbewahrt, doch erhielt ich denselben als Trockenpräparat.

Sehr auffallend erschien zunächst an dem Vogel eine deutlich hellbräunliche Ränderung an den Federn der Unterseite und einzelnen Bürzelfedern, - eine Färbungseigenthümlichkeit, wie sie meines Wissens bisher noch nicht in dem Genus Collocalia beob- 
achtet worden ist. Wo sonst bei bestimmten Species dieser Gruppe eine partielle oder totale helle Coloration gewisser Theile des Gefieders auftritt, zeigt sich vielmehr bekanntlich ausgesprochene Tendenz zum Albesciren: die betreffende helle Färbung ist entweder rein weiss, oder grau in verschiedenen Schattirungen. - Inzwischen habe ich Ursache anzunehmen, dass auch im vorliegenden Falle die befremdende bellbraune Coloration eine lediglich a c cidentelle ist. Wie erwähnt, erhielt ich den Vogel getrocknet; dagegen gingen mir die Eier in einem Fläschchen zu, welches mit einer klaren, aber intensiv röthlich brau n en alcoholischen Flüssigkeit gefüllt war. Ob nun thatsächlich auch bei dem Vogel ein gleiches Conservirungsmaterial in Anwendung gekommen ist, vermag ich augenblicklich nicht zu ermitteln, doch liegt die Vermuthung nahe, dass hierin die Quelle jener abnormen Färbung zu suchen ist. Noch ein anderweitiger Umstand scheint dies fast zur Gewissheit zu machen. Bei Gelegenheit eines kürzlichen Besuchs des Dresdener zool. Museums sah ich unter der dortigen Collection von Salanganen einen Vogel, welcher mit dem in Rede stehenden viel Uebereinstimmendes $\mathrm{zu}$ haben schien und überdies noch dadurch von besonderem Werth für mich war, dass er, nach Ausweis der Etiquette, - gleichfalls von Cebú stammt! Durch gütige Vermittelung des Directors genannten Instituts, Herrn Hofrath Dr. A. B. Meyer, dem ich hierfür sehr zu Dank verpflichtet bin, wurde es mir nachmals ermöglicht, eine unmittelbare Vergleichung beider Vügel vorzunehmen und bin ich dadurch in meiner früheren Auffassung. bestärkt worden. Abgesehen von geringfügiger Verschiedenheit in der Länge der Fliugel und ähnlichen unerheblichen Differenzen, die ich nur als individuelle, bezw. durch Verschiedenheit der Präparationsmethode bedingte, eraciten kann, vermag ich sonstige Unterschiede beider Vergleichsobjecte nur darin zu finden, dass bei dem Dresdener Vogel alle jene Federränder der Unterseite etc. weiss gefärbt sind, welche bei dem andern (jetzt im Mus. Berol.) braun erscheinen. Nach dem oben Bemerkteu bin ich geneigt, hieraus zu schliessen, dass letztere Färbung eine artificielle ist, und beide Individuen der nämlichen Species angebören; es bleibt zu untersuchen: welcher?

Unstreitig zeigen sie, sowohl in ihren plastischen Verhältnissen, wie im allgemeinen Färbungscharakter, die relativ grösste Uebereinstimmung mit $C$. troglodytes Gr., doch fehlt ihnen die scharf abgesetzte weisse Bürzelbinde, welche von allen Autoren für beide 
Geschlechter dieser Species als charakteristișch bezeichnet wird. Bei den wenigen mir vorliegenden Individuen derselben haben die betreffenden Bürzelfedern dunkle Schäfte und ihre weisse Färbung erstreckt sich entweder nur auf die Spitzen (bei den obersten), oder auf die ganze Feder (bei den mittleren), oder endlich auf einen mehr minder beträchtlichen Theil der Basis (bei den untersten). Der Effect ist, nach der natürlichen Anordnung der Federn, ein oben und unten scharf abgesetztes weisses Band, welches durch die feinen dunkeln Linien der Schäfte kaum unterbrochen erscheint. Dieses Band feblt den Cebú-Vögeln durchaus; nur an einzelnen, unregelmässig theils höher, theils tiefer stehenden Bürzelfedern finden sich schmale helle (weisse) Säume. Dies nur als eine Altersverschiedenheit, bezw. Jugendcharakter deuten zu sollen, scheint mir nicht wohl angängig, da wenigstens der eine Vogel beim F ortpflanzungs geschäft getroffen wurde. Uebrigens zeigt auch der Nestbau (worüber unten) wesentliche Abweichung von dem der C. troglodytes. - Ausser dieser Species dürfte nur noch C. Linchi Horsf, zur Vergleichung in Betracht kommen. Abgesehen jedoch von merklichen Unterschieden der plastischen Verhältnisse, insbesondere bedeutenderer absoluter und relativer Länge des Flügels der Cebú-Salangane, fehlt bei Linchi die weisse Ränderung einiger Bürzelfedern, Kinn und Kehle sind bei dieser einfarbig mausgrau.

In der Erwartung, dass beim Eingange des mir in Aussicht gestellten weiteren Materials sich diese Unterschiede als constant erweisen werden, folgt hier die Diagnose der Art:

Oberseite russfarben, mit dunkelblauem Metallschimmer auf Scheitel-, Flügel- und Steuerfedern; einzelne Bürzelfedern schmal weiss gesäumt.

Unterseite mausgrau, mit weisslichen Federkanten, welche vom Kinn nach abwärts an Breite zunehmen. Unterseite der Steuerfedern dunkelgrau, nach der Basis hin allmählich bedeutend heller gefärbt.

$\begin{array}{lr}\text { Totallänge: } & \text { ca. } 85 \mathrm{Mm} \\ \text { Flügel : } & 94-96 " \\ \text { Schwanz: } & 40 " \\ \text { Schnabel : } & 3 \% \\ \text { Tarsus: } & 9 "\end{array}$

Was die Nistweise betrifft, so ähneln die vorliegenden 4 Nester sowohl denen, welche Bernstein (Jahrg. 1859, S. 113 d. J.) von 
seiner javanischen „C. fuciphaga Thunb."*) beschreibt, als auch solchen der C. spodiopygia (Peale), welche das Museum Godeffroy von Niuafou (Tonga-Inseln) empfing; sie gehören also zu den nicht essbaren und haben die Gestalt halbrunder Körbchen, welche mit ihrer hiuteren, flacheren Wand am Felsen angeleimt waren, während die vordere stark gewölbt vorspringt. Die seitlichen flügelförmigen Ansätze der hinteren Nestwand, wie sie bei anderen Arten (nidifica, troglodytes, zuweilen auch bei spodipygia) bemerkt werden, fehlen hier ganz, oder sind doch nur schwach angedeutet. Das Baumaterial besteht aus feineren oder gröberen biegsamen Pflanzentheilen: Halmen, Stengeln, Baststreifen, Manilafasern u. dergl., welche entweder einfach parallel über einander gelagert oder inniger mit einander verflochten sind; im ersteren Falle hat der Vogel das Material mit einem mehr oder minder starken Speichelüberzuge fest zusammengeleimt. Mit dem gleichen Klebestoff ist die innere Höhlung des Nestes in dünnerer oder dickerer Auftragung überzogen, und relativ besonders reichlich findet sich die Leimsubstanz zur Festigung des freien vorderen Randes sowie der hinteren Nestfläche verwendet. Der Querdurchmesser der Nester beträgt etwa 7, der gerade 5, die Höhe an der hinteren Seite 5, vorn in der Mitte $4 \mathrm{Cm}$. - Sehr verschieden hiervon sind Nester der C. troglodytes, welche von Herrn Dr. Schadenberg auf Luzon gesanmelt wurden. Bei beträchtlich geringerer Grösse, bestehen dieselben durchweg: aus eiuem durchbrochenen Netzwerk erhärteter Speichelleimfäden, zwischen welchen nur an der vorderen Nestwand einzelne Halme und Blattreste eingeflochten sind. Diese Nester bilden sonach den Uebergang zu den bekannten, lediglich aus der schaalenartig geformten Speichelcolla bestehenden der $C$. nidifica.

Die Eier entsprechen dem Typus aller Verwandten; sie sind rein weiss, glattschaalig, aber nur schwach glänzend; die Gestalt

*) C. fuciphaga (Thunb.) hat das Missgeschick, sehr verschieden gedeutet zu werden. Während $z \cdot B$. Walden (Ibis 1×74, p. 135) diese Art mit Linchi Horsf. identificirt, glaubt ein anderer bedeutender Forscher, Salvadori (Osserv. d. Gen. Collocalia - Atti d. Aead. d. Torino 1880), dieselbe mit $C$. nidifica, G. R. Gray für synonym balten zu müssen. Hinsichtlich der von Bernstein (l. c.) als fuciphaga 'Thunt. erwähnten javanischen Art dürfte es m. E., nach der beigefügten Beschreibung des Vogels, keinem Zweifel unterliegen, dass dieselbe mit Linchi Horsf. zusammenfällt, obwohl letztere Species von dem Genannten (l. c., S, 119, sub 4) noch gesondert aufgefüht wird. 
ist walzenförmig, mit wenig verjüngter Spitze. Maasse: $16-17+$ 10-11 $\mathrm{Mm}$. Zar Vergleichung füge ich hier die Dimensionen der Eier einiger verwandten Arten, die ich zu untersuchen vermochte, bei:

C. nidifica G. R. Gray, - ex India - 20-21 + 13,5-14 Mm.

"spodiopygia (Peale) - ex Polynesia - 17,6-19+12-12,7 "

" Linehi Horsf. - ex Nov.Britannia - 16-17,3+10,7+11 "

19. Lyncornis macrotis (Vig.), 2 Vögel.

20. Caprimulgus griseatus G. R. Gray, - ?

Ein unter der tagalischen Bezeichnung „Gandara" (?) vorliegendes Caprimulgiden-Ei möchte ich fast mit Bestimmtheit, schon nach Maassgabe seiner Grössenverhältnisse, der genannten Species zusprechen. Abgesehen von diesem Beurtheilungsmomente kommt noch in Betracht, dass es mit dem Typus der Eier von C. albonotatus und macrourus, welche mit manillensis, der zweiten auf Luzon vorkommenden Art, zu einer Gruppe gehören, wenig Aehnlichkeit zeigt, wohl aber mit dem Ei von $C$. affinis, einem sehr nahen Verwandten von griseatus, welches König-Warthausen beschreibt, auf das Genaueste übereinstimmt (vergl. Jahrg. 1868, S. 273 d. J.).

Die Form ist gleichhälftig oval, mässig gestreckt; die Schaale glatt, feinkörnig, glänzend, mit sparsamen gerundeten Poren; Grundfarbe weisslich, mit schwachem Stich ins Gelbliche. Die Zeichnung besteht aus ziemlich kleinen violetgranen Schaalenflecken und bräunlichen Oberfleckchen, welche das Ei ziemlich gleichmässig, aber nicht dicht, bedecken. Maasse: $26,4+19 \mathrm{Mm}$; Gewicht: $36 \mathrm{Cg}$.

21. Dasylophus supereiliosus Cuv., 2 Vögel, ad. (Iris, nach Notiz des Sammlers, gelb). Der Schnabel des einen Exemplars schwach grünlich weiss, an der Spitze gelblich; bei dem andern, sonst nicht versehiedenen Vogel Oberschnabel hornbraun, Spitze desselben und Unterschnabel schmutzig grün.

22. Centrococcyx viridis (Scop.), 1 Vogel, ad. („Iris roth, Totallänge 40, Umfang über die Brust $16 \mathrm{Cm}$.", nach beiliegender Notiz).

23. Buceros hydrocorax Lin., tagalisch "Calao", 2 Vögel, ad. („Iris braun").

24. Penelopides Manillae (Bodd.), $3 \delta$ ad., $2 q$ ad. Zur Vergleichung mit den von Walden ('Tr.Z. S. IX., p. 169) angcgebenen Maassen folgen hier die der vorliegenden Vögel: 


\begin{tabular}{|c|c|c|c|c|c|c|}
\hline $\mathrm{No}$ & . & $\mathrm{ad}$ & Schnabel. & Flügel. & $\begin{array}{c}\text { Schwan } \\
290\end{array}$ & $\mathrm{Mm}$ \\
\hline " & $2, \delta$ & " & 84 & 230 & 215 & , \\
\hline " & $3, \delta$ & $n$ & 83 & 227 & 200 & $n$ \\
\hline "s & 4,9 & $"$ & 70 & 220 & 200 & \# \\
\hline 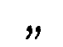 & 5,9 & $n$ & 67 & 220 & 200 & $n$ \\
\hline
\end{tabular}

Die Färbung des Gefieders stimmt im Allgemeinen mit der von dem genannten Autor (l. c.) gegebenen Beschreibung ïberein. Bei allen vorliegenden Stücken ist das Mittelband des Schwanzes an beiden äusseren Paaren der. Steuerfedern hell-isabellfarbig. Bei No. 1 geht diese Färbung an den folgenden Paaren stufenwe ise in die tiefe Rostfarbe der mittelsten über. Bei den $q q$ ist das in Rede stehende Band merklich schmaler als bei den $\delta$ o und erstreckt sich auch nicht über die ganze Breite der Innenfahne des äussersten Paars der Steuerfedern, sondern bildet daselbst nur einen den Federschaft mit seiner Spitze nicht erreichenden Keilfleck.

25. Caratua haematuropygia (Müll.), 2 Vögel.

26. Tanygnathys lucionensis (Lin.), 2 Vögel.

No. 1. Vorderkopf grün; Scheitel, Hinterkopf und Nacken mit türkisblauen Federspitzen; Unterrücken fast rein blau; Bürzel mehr grün.

No. 2. Scheitel grün; Hinterkopf und Nacken sparsamer und mit blasserem Blau gezeichnet; sonst mit vorigem übereinstimmend.

27. Spilornis holospilus (Vig.), 3 Vögel (2 ad., 1 juv.) - sämmtlich im Mas. Berol. -

28. Pernis ptilorhynchus (Tem.), 1 Vogel, aus dem District Baatan, nördlich von Manila. (Mus. Berol.) Neu für Luzon.

29. Hierax sericeus (Kittl.), 1 Vogel; ohne Superciliarstreifen, Wangen weiss.

30. Carpophaga paulina Bp., 1 Vogel, Distr. Baatan.

Stirn, Kinn und Kehle weisslich; Scheitel schwach rötblichgrau; Hinterkopf blaugrau; Nacken glänzend fuchsroth; übrige Oberseite goldig grün. Schwung - und Steuerfedern glänzend dunkelblau, mit grünem Schiller an den Rändern; Schultern wie der Hinterkopf; Hals, Brust und Bauch grau,-intensiv weinroth überlaufen. Untere Schwanzdecken dunkel rothbraun. Innenseite der Steuer- und Schwungfedern hell bräunlich grau.

$$
\begin{aligned}
& \text { Flügel, } 220 \mathrm{Mm} . \\
& \text { Schwanz, } 145 \%
\end{aligned}
$$




\section{Schnabel, $26 \mathrm{Mm}$.}

\section{Tarsus 30 "}

Von Celebes-Exemplaren erscheint der Vogel demnach nur durch etwas geringere Maasse und vielleicht etwas hellere Färbung des Oberkopfes verschieden. Neu für das ganze Gebiet.

31. Carpophaga aenea (Linn.), 2 Vögel, Distr. Baatan. Kopf hell bläulich grau, Nacken von etwas dunklerer Schattirung; Brust und Bauch wie der Kopf, aber gleichmässig schwach weinroth überlaufen.

32. Carpophaga nuchalis Cab., Journ. f. Orn. 1882 , p. 126. 1 Vogel, Distr. Baatan. (Mus. Berol.)

Durch beschränkten, stumpf dunkelkastanienbraunen Nackenfleck deutlich, sowohl von der vorigen Species, als von paulina unterschieden, weshalb ich mich von vornherein nicht entschliessen konnte, den Vogel mit einer derselben zu identificiren. Erst später wurde ich durch eine Notiz Walden's (Tr. Z. S. IX., p. 215) darauf aufmerksam, dass bereits Schlegel, auf Grund eines einzelnen, durch Gevers von Luzon erhaltenen Exemplares eine ,forme intermédiaire" unterschieden hat. Seine Beschreibung des Vogels im Vergleich mit paulina: „la tache de la nuque beaucoup plus restreinte et teinte d'nn ronge de cuivre très foncé et terne" (Nederl. Tijdschr. Dierk. 1866, p. 201) passt durchaus auch auf das vorliegende, nunmehr also $\mathrm{z}$ weite Exemplar von demselben Fundorte.

33. Osmotreron axillaris, Bp., 1 Vogel. Untere Schwanzdecken grünlich gefleckt ( $q$ ?).

34. Gallus bankiva, Tem., 1 ㅇ.

35. Turnix ocellata (Scop.). 1 ㅇ.

36. Ardea purpurea, Lin., 1 Vogel.

37. Ardetia cinnamomea (Gmel.), 4 ad., 1 juv.

Zwei Eier, die unter der Bezeichnung „Matambajam“ (Wasserhuhn) vorliegen, möchte ich dieser Species zuschreiben, da sie nicht wohl einem anderen in Betracht kommenden Sumpf- oder Schwimmvogel angehören können und mit einem javanischen $\mathrm{Ei}$ des Zimmtreihers, bis auf etwas bedeutendere Grösse, ubereinstimmen. Form fast gleichhälftig oval; Färbung schmutzigweiss, mit einem Stich in's Bläulichgrüne; Schaalenoberfäche matt, mässig feinkörnig, mit ziemlich zahlreichen gerundeten Poren. Maasse: $35,3+28$ und $36+36,5 \mathrm{Mm}$.; Gewicht: 73 und $69 \mathrm{Cg}$. 
38. Butio (Gorsachins) Kutteri Cab., Journ. f. Orn. 1881, pag. 425. 1 Vogel ad., Distr. Baatan. (Mus. Berol.)

39. Hypotaenidia torquata (Lin.), tagalisch "Ticlin", 1 o ad., 2 Eier. Letztere gleichen denen aller Verwandten; insbesondere solchen ron Rallus pectoralis, Forst., bis auf etwas geringere Grösse. Form gedrungen eiförmig, nit wenig verjüngter Höbe und stark abgerundeter Basis; Schaale mässig glänzend, feinkörnig, mit sparsamen, flachen Poren. Grundfarbe röthlich isabellgelb. Die Zeichnung besteht in violetgrauen Schaalenfleckchen und intensiv kastanienbraunen, gerundeten oder etwas in die Lünge gezogenen, scharf contourirten Spritzflecken und Punktell. Maasse: $36,9+27,5$ und $36,7+27 \mathrm{Mm}$.; Gewicht: 129 und $126 \mathrm{Cg}$. Berol.)

40. Gallicrex cinerea (Gm.), 1 ठ , Distr. Baatan. (Museum

41. Anas luzonica, Fras., 2 Vögel.

\title{
Oruithologische Notizen aus Central-Afrika. Von Dr. R. Böhm. \\ Mitglied der deutschen Expedition nach Ost-Afrika.
}

\author{
An Dr. Reichenow.
}

IKakoma, Mai 1881.

Im Folgenden übersende ich Ihnen, als Ergänzung der von Zanzibar, Tabora und Kakoma abgeschickten ornitbologischen Sammlungen, einige meinem Journale entnommene biologische Notizen.

Nie ist mehr, als hier im aequatorialen Afrika, die Ueberzeugung in mir wach gewesen, dass der Vogel in seiner Eigenart nur im Zusammenhang und in Beziehung zu der ganzen ihn umgebenden Natur verständlich ist, zu den meteorologischen und klimatischen Verhältnissen nicht minder, wie zu der Bodenformation, der Flora, namentlich in pflanzengeographischer Hinsicht und der übrigen Fauna des Landes. Ein derartiges, zusammenhängendes Bild der hiesigen Ornis zu geben, muss ich mir aber für spätere Zeiten vorbehalten. Hier nur Einiges von dem zu einem solchen $Z_{\text {wecke }}$ bisher gesammelten Rohmaterial.

1. Struthio camelus L.

$$
\text { STRUTHIONIDAE. }
$$

Der Strauss ist besonders in den theils ganz offenen, theils mit niedrigem Buschwald bestandenen Hochebenen von Ugog*0 nicht 